\title{
Amazing structure of respirasome: unveiling the secrets of cell respiration
}

\author{
Runyu Guo, Jinke Gu, Meng Wu, Maojun Yang ${ }^{\bowtie}$ \\ Ministry of Education Key Laboratory of Protein Science, Tsinghua-Peking Joint Center for Life Sciences, Beijing Advanced \\ Innovation Center for Structural Biology, School of Life Sciences, Tsinghua University, Beijing 100084, China \\ $\bowtie$ Correspondence: maojunyang@tsinghua.edu.cn (M. Yang) \\ Received September 15, 2016 Accepted September 28, 2016
}

\begin{abstract}
Respirasome, a huge molecular machine that carries out cellular respiration, has gained growing attention since its discovery, because respiration is the most indispensable biological process in almost all living creatures. The concept of respirasome has renewed our understanding of the respiratory chain organization, and most recently, the structure of respirasome solved by Yang's group from Tsinghua University (Gu et al. Nature 237(7622):639-643, 2016) firstly presented the detailed interactions within this huge molecular machine, and provided important information for drug design and screening. However, the study of cellular respiration went through a long history. Here, we briefly showed the detoured history of respiratory chain investigation, and then described the amazing structure of respirasome.
\end{abstract}

KEYWORDS respirasome structure, supercomplexes organization, cellular respiration, respiratory complexes, megacomplex

\section{INTRODUCTION}

Energy generation and consumption are the essential foundation of every biological process. Unlike gas or electricity used by industry, energy conversions occurred in living organisms are gentle and efficient, therefore much more complicated. According to the sources of energy flows, energy conversions can be divided into two classes. One class captures energy from light and fixes solar energy into various organic compounds, which is called photosynthesis; and the other class releases energy from organic compounds and generates the high-energy molecule ATP later consumed by virtually all other biological pathways, which is called respiration.
Photosynthesis are performed mainly in the lower grade photosynthetic bacteria, algae, and chloroplasts in higher plants (Dudkina et al., 2015). They harness solar energy and produce organic compounds containing stable chemical energy, which is the energy source of almost all animals. The processes of photosynthesis can be separated into two parts, light reaction and dark reaction. In chloroplasts, light reaction occurs on the membrane of thylakoids. Two types of protein systems, photosystem I (PSI) and photosystem II (PSII), participate in the capture of photons and generation of high-energy electrons (Dekker and Boekema 2005; Kouril et al., 2012). Those generated electrons can be transferred in two modes of electron flow: linear electron flow (LEF) and cyclic electron flow (CEF). In LEF, both PSI and PSII are involved, while only PSI participates in CEF. Both ATP and NADPH are produced by LEF, but only ATP can be produced by CEF (Johnson, 2011; Rochaix 2014). As a result, the balance between PSI and PSIl can influence the final ratio of ATP/NADPH, which is important for dark reaction. Dark reaction occurs in chloroplast stroma, where $\mathrm{CO}_{2}$ is fixed and carbohydrates are produced (Livingston et al., 2010).

Respiration takes place in almost every living creature, and in higher organisms the executor of respiration becomes more and more sophisticated. In mammals, respiration is carried out in mitochondria. Through various catabolism pathways, carbohydrates, lipids, cholesterol, and some kinds of amino acids are catalyzed into small electron donor molecules, such as NADH and FADH, and subsequently these electron donors enter the respiratory chain located on the inner mitochondrial membrane (IMM) (Genova and Lenaz 2014). Via the respiratory chain, electrons are finally transferred to oxygen, and the energy released this way is used to pump protons from mitochondrial matrix (MM) to intermembrane space (IMS), thus forming the electrochemical gradient of proton. Ultimately, ATP synthase (complex V, 
CV) uses the energy within this gradient to synthesis ATP, and all these procedures together are termed as respiration (Mitchell 1961). The recognition of respiratory chain complexes and analyzing of their organization went through a long and detoured history.

Currently, it is believed that four classes of protein complexes which are relatively independent in function constitute the respiratory chain. They are complex I (NADH: ubiquinone oxidoreductase, $\mathrm{Cl}$ ), II (succinate: ubiquinone oxidoreductase, CII), III (ubiquinone: cytochrome c oxidoreductase or bc1 complex, CIII), and IV (cytochrome c oxidase, CIV). According to latest structural researches, these four classes of protein complexes are very likely to interact with each other and forming a higher-order structure, respiratory supercomplex (Vartak et al., 2013). Different combination of individual $\mathrm{CI}$-CIV can produce different types of supercomplexes, and the supercomplexes that can fully perform the respiration reaction (consuming electron donors and oxygen while generating water molecules) are also termed as respirasome. (Enriquez 2016). Since respiration is irreplaceable in almost all living creatures, mutations occurred within respiratory chain complexes can lead to various severe physiological defects directly or indirectly. In the following sections, we'll first briefly describe the research history of respiratory chain, and then analyze the structure of respirasome that are determined in our latest work.

\section{RECOGNITION OF RESPIRATORY CHAIN COMPLEXES IN HISTORY}

Discovery of basic respiratory chain elements in the early stage

From the early days of the 20th century, scientists successively uncovered many kinds of redox enzymes and prosthetic groups responsible for electron transfer. In 1900, Michaelis from America found mitochondria could be stained by Janus Green B. (Ernster and Schatz 1981). The staining ability of Janus Green B is dependent on the its redox state, therefore it was speculated later that mitochondria was the place where cellular respiration occurred. A couple of years later, Warburg coarsely extracted mitochondria from cavy hepatocyte for the first time in 1912, and identified several enzymes capable of conducting redox reactions, thus denominating them as respiratory enzymes (Ernster and Schatz 1981). Till the 1940s, Hogeboom firstly isolated the morphologically well-preserved mitochondria and demonstrated the location of succinoxidase and cytochrome c oxidase (Ernster and Schatz 1981). In the 1950s, the coupling sites of respiratory chain were largely recognized (Ernster and Schatz 1981). Gradually, scientists realized that cell respiration was not performed by some specific types of enzymes, but by a large series of enzymes and prosthetic groups forming the respiratory chain (Ernster and Schatz 1981).
Till the 1960s, substantially all the prosthetic groups in the respiratory chain were identified, and their precise order in the chain was also determined through methods like measuring the standard redox potential of each element, comparing the oxidation state of each element when the reaction reaches balance, and using inhibitors aimed at specific electron acceptors. (Ernster and Schatz 1981). Instead of reacting separately, the enzymes and prosthetic groups assemble into functional modules performing energy transducing roles coordinately. Totally 4 functional modules were purified and reconstructed by Hatefi et al., till 1962, termed Cl-CIV. (Hatefi et al., 1962) From that time on, work by Green, Tzagoloff and Hackenbrock in the subsequent twenty years established the fluid model of the IMM organization, (Green and Tzagoloff 1966; Hochli and Hackenbrock 1976) where all redox components are independent diffusible particles with the small electron carriers shuttling between the huge respiratory complexes I-IV, hence electron transport is considered a multicollisional, obstructed and longrange diffusional process (Hackenbrock et al., 1986) Due to lack of structural information, the mechanism of protonpumping and electron-transfer within these complexes were largely unknown back then (Fig. 1).

\section{Structure determination of individual respiratory complexes from the 1990 s}

From 1995 to 2003, structures of CIV isolated from prokaryotic organisms and mammals were sequentially determined, and its function mechanism became more and more clear (Iwata et al., 1995; Tsukihara et al., 1995, 1996, 2003; Ostermeier et al., 1997; Kannt et al., 1998; Yoshikawa et al., 1998). CIV has three core subunits encoded by mitochondrial DNA, called SU1, SU2 and SU3. SU1 has two heme centers (heme $a$ and heme $a_{3}$ ) and one $\mathrm{Cu}$ center (CuB). Heme $a_{3}$ and CuB together form a binuclear center, which is the site for oxygen reduction. SU2 contains a second $\mathrm{Cu}$ center (CuA) which comprises of two $\mathrm{Cu}$ atoms and accepts the electrons delivered from cytochrome c. SU3 has no redox centers but can interact with SU1. The other 11 subunits of CIV were considered to stabilize the whole enzyme. Electrons from cytochrome $c$ were first transferred to $\mathrm{CuA}$, then to the low spin heme a, subsequently to the high spin heme $a_{3}$, and finally to [Fe-Cu] center where $\mathrm{O}_{2}$ was captured and reduced. There exist two kinds of proton pathways. Through one pathway protons were delivered to oxygen reduction center and fixed into water molecules, and by the other pathway protons were pumped from matrix to IMS directly through conformational change caused by heme a reaction. The first pathway is called K/D-pathway, through which protons from matrix were transferred via Lys354 or Asp124 and a series of hydrogen bonds to heme $a_{3}$ and [FeCu] center. (Tsukihara et al., 1996; Yoshikawa et al., 1998). The second pathway is called H-pathway, where His413 at the matrix side and Asp 51 at the IMS side played important 


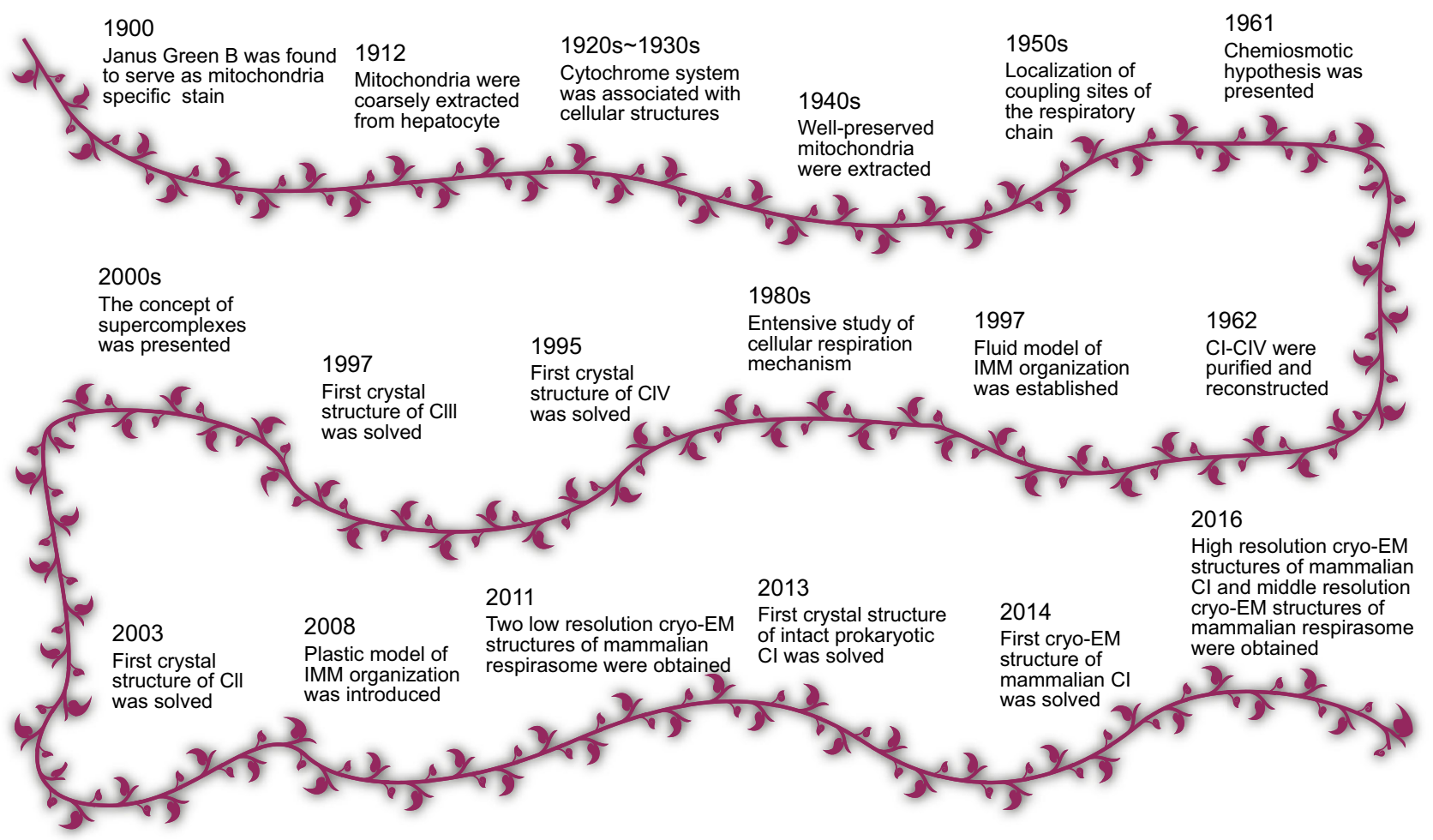

Figure 1. Brief research history of the respiratory chain.

roles. Before heme a was reduced, protons from matrix could access Asp51 via a net of hydrogen bonds and one peptide bond inside the hydrogen bonds network can prevent reverse transfer. When electrons delivered to heme a, the conformational change of CIV occurred, and protonated Asp51 was exposed to the IMS side, where the proton can be released through a set of hydrogen bonds (Tsukihara et al., 2003; Kaila et al., 2011). With every one electron transferred from cytochrome $c$ to oxygen molecule, one proton is transferred to the [Fe-Cu] site and another proton is translocated from matrix side to IMS side. But the reason why there are two pathways ( $K$ and $D$ pathways) responsible for oxygen reduction is not clear, and the functional difference between these two pathways is not know either (Fig. 1).

Structures of CIII have been obtained from bovine, chicken and rabbit heart since 1997, (Xia et al., 1997; Iwata et al., 1998) and till 2003 subsequent studies even got CIII crystal structures bond with either cytochrome $\mathrm{c}$ or inhibitors (Zhang et al., 1998; Gao et al., 2002, 2003; Lange and Hunte 2002; Palsdottir et al., 2003). Many hypotheses have been proposed to describe the mechanism of proton translocation and electron transfer within CIII, among which the Q-cycle theory has the largest influence. Every CIII has three conserved subunits with active redox centers. They are cytochrome $\mathrm{b}$ (SU3) containing heme $b_{H}$ and heme $b_{L}$, cytochrome $c_{1}$ (SU4) containing heme $c_{1}$, and ISP (SU5, Rieske protein, iron-sulfer cluster binding protein) containing one [2Fe-2S] cluster. These 3 subunits form the core of CIII and are responsible for the electron transfer and redoxcoupled proton translocation function, with the other 8 accessory subunits holding them together. According to the $\mathrm{Q}$-cycle theory, there exist two kinds of $Q$ binding sites, including $Q_{0}$ site, which is near the IMS side and $\mathrm{QH}_{2}$ is oxidized to $Q$ by heme $c_{1}$, and $Q_{i}$ site, which is near the matrix side and $\mathrm{Q}$ is reduced to $\mathrm{QH}_{2}$ by heme $b_{H}$. Three configurations of $\mathrm{CIII}$ were also classified, according to the distance between ISP, heme $b_{L}$, and heme $c_{1}$. In b position state, ISP is close to heme $b_{L}$, while in c position state, ISP is close to heme $c_{1}$. In the third state, int state, ISP is relatively far from both heme $b_{L}$ and heme $c_{1}$ (Zhang et al., 1998). When no $Q$ or $\mathrm{QH}_{2}$ were bond to CIII, ISP is in the int state. After $\mathrm{QH}_{2}$ binded to $\mathrm{Q}_{\mathrm{o}}$ site, $\mathrm{QH}_{2}$ is deprotonated to $\mathrm{QH}$ - and ISP was moved to the $b$ position state, in the meanwhile $\mathrm{QH}^{-}$ delivered one electron to ISP to form semiquinone. After the semiquinone bonded to [2Fe-2S] cluster in ISP moved to the b position sate, the semiquinone delivered the second electron directly to heme $b_{L}$, and the formed $Q$ was released from the $[2 \mathrm{Fe}-2 \mathrm{~S}]$ cluster. Then, the reduced ISP moved to the $c$ position sate, where the first electron rapidly transferred from the [2Fe-2S] cluster to heme $c_{1}$, then to cytochrome $c$. The electron delivered to heme $b_{L}$ was further 
transferred to heme $b_{H}$ to reduce $\mathrm{Q}$ to $\mathrm{QH}_{2}$ at Qi site. After the electron transfer, ISP moved back to its int state, preparing to react with the next $\mathrm{QH}_{2}$. In one $\mathrm{Q}$ cycle, one $\mathrm{QH}_{2}$ binds to $\mathrm{Q}_{\mathrm{o}}$ site, releases two protons to IMS, and delivers one electron to cytochrome $c$ and one electron to $Q$ at $Q_{i}$ site, where two electrons are needed to reduce $Q$ to $\mathrm{QH}_{2}$ (Iwata et al., 1998). So, in one $\mathrm{Q}$ cycle only one electron is delivered to one cytochrome $c$ and one proton is consumed from matrix, but two protons are released to IMS. The reason why only one of the two electrons from $\mathrm{QH}_{2}$ can be delivered to cytochromec is not interpreted, which seems very inefficient (Fig. 1).

The next breakthrough happened in 2003, when the crystal structure of prokaryotic version Cll was solved (Yankovskaya et al., 2003). 2 years later, CIl was purified from porcine and avian hearts, with the resolution of $2.4 \AA$ and 2.1 $\AA$ respectively (Sun et al., 2005; Huang et al., 2006). Eukaryotic Cll consists of a soluble heterodimer domain and an integral transmembrane region. The soluble domain contains Fp and Ip subunits, with the Fp subunit binding the FAD cofactor and the Ip subunit containing 3 Fe-S clusters. The transmembrane domain also comprises two subunits, CybL and CybS, with one heme b buried inside. To be brief, in the Fp subunit succinate is dehydrogenated to fumarate, with two electrons being extracted by FAD and transferred through three Fe-S clusters to the UQ binding site formed by IP, CybL and CybS (Sun et al., 2005). Two protons are generated at the matrix side by succinate oxidation and two other protons consumed at the IMS side by ubiquinone reduction, thus with no net proton translocated. $\mathrm{Cll}$ is considered as a branch of the respiratory chain, and there exist many Cll-like auxiliary electron donors, which participate in different metabolism pathways, including flavoprotein: ubiquinone oxidoreductase in $\beta$-oxidation, dihydroorate dehydrogenase in pyrimidine synthesis, choline dehydrogenase in glycine metabolism, sulfide:ubiquinone reductase in sulfur and seleno-amino acid metabolism, proline dehydrogenase in arginine and proline metabolism, and glycerol-3-phosphate dehydrogenase in shuttling reducing equivalents from cytoplasm (Enriquez, 2016; Lenaz and Genova 2010) (Fig. 1).

From 2006 to 2013, Sazanov's group gradually solved the structure of the intact $\mathrm{Cl}$ from prokaryotic cells to high resolution, (Sazanov and Hinchliffe, 2006; Baranova et al., 2007; Sazanov, 2007; Morgan and Sazanov, 2008; Berrisford and Sazanov, 2009; Efremov et al., 2010; Efremov and Sazanov, 2011; Baradaran et al., 2013) and in 2014, Hirst's group determined the structure of bovine $\mathrm{Cl}$ at a medium resolution (Vinothkumar et al., 2014). Recently in 2016, Hirst's group pushed the resolution of their bovine $\mathrm{Cl}$ structure to $4.2 \AA$, (Zhu et al., 2016) and Sazanov's group got a $3.9 \AA$ resolution structure of $\mathrm{Cl}$ from ovine heart mitochondria (Fiedorczuk et al., 2016) Moreover, via sub-region refinement, our group can also get a $3.96 \AA$ density map of $\mathrm{Cl}$ from porcine mitochondria ( $\mathrm{Gu}$ et al., 2016). Mammalian $\mathrm{Cl}$ has 45 subunits in total, among which 14 are core subunits encoded by mitochondrial DNA, and the other 31 are supernumerary subunits. All these structures show the intact structure of $\mathrm{Cl}$ to be L-shaped, with a matrix arm and a membrane arm. The core subunits of $\mathrm{Cl}$ form a foot-like structure slipping into a sandal-like structure composed by the circling supernumerary subunits (Gu et al., 2016). The matrix arm containing NDUFV1, NDUFV2, NDUFS1, NDUFS2, NDUFS3, NDUFS7 and NDUFS8 accommodates FMN molecule and the Fe-S clusters, which oxidizes NADH and transfers electrons to the $Q$ reaction site. The proximal membrane subunits ND1, ND2, ND3, ND4L and ND6 catalyze the reduction of ubiquinone and couple the energy released from $Q$ reduction to proton pumping through longrange conformational change. The distal membrane subunits ND4 and ND5 are antiporter-like proteins and perform the duty of proton pumping. ND2 is another antiporter-like protein, and together with the presumed channel formed by ND1 and ND4L, Cl have four proton channels in total, but accurate proton pumping pathways are not identified yet and convincing coupling mechanism is lacking. (Fiedorczuk et al., 2016). The supernumerary subunits of $\mathrm{Cl}$ were considered to stabilize $\mathrm{Cl}$ and facilitate $\mathrm{Cl}$ assembly. (Zhu et al., 2016) (Fig. 1).

\section{RESPIRATORY SUPERCOMPLEX MODEL}

\section{Identification of respiratory supercomplexes}

In 2000, via Blue-Native PAGE (BNPG), supercomplexes (SCs) were identified and an exciting new perspective into the organization of OXPHOS system was provided by Schägger (Schagger and Pfeiffer, 2000). BNPG is suitable for detecting molecules with large molecular weight and maintains the enzyme activity (Wittig et al., 2006). After digitonin solubilization of bovine mitochondria, the supernatant was applied to glucose-gradient ultracentrifugation. Then each layer from the centrifuge tube was used as a sample to run BNPG. Above the $1 \mathrm{MDa}$ bands representing $\mathrm{Cl}$, many larger molecular weight bands still exist. Together with results from second dimensional SDS page and Western blot, these bands were identified to contain subunits from $\mathrm{Cl}, \mathrm{CIII}$ and CIV. Very soon, many groups used similar procedure to identify supercomplexes from bacteria, yeast, plant and mouse, and different patterns of supercomplex composition were reported, (Schagger and Pfeiffer, 2000, 2001 Schagger, 2001; Schagger, 2002; Pfeiffer et al., 2003; Wittig et al., 2006; Nubel et al., 2009; Zhang et al., 2005; Heinemeyer et al., 2007; Stuart, 2008; Daoud et al., 2012; Ramirez-Aguilar et al., 2011; Dudkina et al., 2006; Eubel et al., 2004; Eubel et al., 2003; Gomez et al., 2009; AcinPerez et al., 2008; Lapuente-Brun et al., 2013; Wenz et al., 2009; Stroh et al., 2004). Technically, supercomplexes that are able to fulfill the respiration activity are also termed respirasomes.

Schägger proposed that the OXPHOS complexes are not randomly scattered in the IMM, but assembled into higher- 
order structures, which is called the solid model (Schagger and Pfeiffer, 2000; Chance et al., 1963). In this model, Cl, CIII and CIV can be assembled into different forms of supercomplexes, including $I_{1} I I_{2} I V_{1}, I_{1} I I_{2} I V_{2}, I_{1} I I_{2} I V_{4}, I I_{2} I V_{1}$, and $I I I_{2} I V_{2}$. Those detected free forms of respiratory complexes were interpreted as assembly intermediates (Fig. 1).

Since the definition of SC, various evidence suggesting the advantages of forming SC has been accumulated. The advantages appear in four aspects (Dudkina et al., 2015; Genova and Lenaz, 2014; Enriquez, 2016; Lenaz and Genova, 2010; Cogliati et al., 2016; Moreno-Loshuertos and Enriquez, 2016; Genova, 2014; Sazanov, 2015; Liao et al., 2015): (1) CIII and CIV integrated onto Cl to form SC can stabilize $\mathrm{Cl}$. (2) SCs produce much less ROS (reactive oxygen species). (3) The catalytic activity of individual components is higher in SCs. (4) Through substrate channeling, the efficiency of electron transfer is elevated in SCs. In 2002, Lamantea and his colleagues discovered that some CIII abnormalities not only disrupt the activity of CIII, but also hamper the proper function of $\mathrm{Cl}$ (Lamantea et al., 2002). In 2004, Acìn-Perèzand his colleagues reported that when $\mathrm{CIII}$ is ablated, $\mathrm{Cl}$ is prone to degradation in mitochondria (Acin-Perez et al., 2004). Subsequently, other groups confirmed this phenomenon, and lack of cytochrome c was found to disrupt the assembly and stability of $\mathrm{Cl}$ and CIV (Vempati et al., 2009). In 2012, Moreno and his colleagues found that partially assembled $\mathrm{Cl}$ can interact with $\mathrm{CIII}$ and $\mathrm{CIV}$, and they claimed that completion of $\mathrm{Cl}$ assembly requires interaction with CIII and CIV (Moreno-Lastres et al., 2012; Diaz et al., 2006). The prevention of ROS formation is another advantage of $\mathrm{Cl}$ and CIII assembling into SC. The ROS generating sites are considered to be $\mathrm{FMN}$ and $\mathrm{N} 2$ in $\mathrm{Cl}$ and $\mathrm{Q}_{0}$ site in CIII, where semiquinone can be formed and oxygen is accessible. (Genova, 2014) In 2013, Maranzana and his colleagues reported that in both purified bovine mitochondria and reconstituted liposomal preparations, the disruption of SC strongly enhance the generation of superoxide and $\mathrm{H}_{2} \mathrm{O}_{2}$ by $\mathrm{Cl}$. (Maranzana et al., 2013) The structural explanation of SC preventing ROS formation is still lacking. There are also some implications that SCs can enhance the catalytic activity of their component complexes. In 2006, Schägger and his colleagues found that the activity of $\mathrm{Cl}$ and $\mathrm{CIII}$ is higher in $\mathrm{SCl}_{1} \mathrm{II}_{2} \mathrm{IV}_{1}$ compared to the SC lacking the terminal oxidase, $\mathrm{I}_{1} \mathrm{III}_{2}$ (Schafer et al., 2006).

Evidence for substrate channeling in SC have been growing. Interaction between $\mathrm{Cl}$ and $\mathrm{CIII}$ can cause segmentation of $Q$ pool. Ubiquinone functions as the center of electron transfer to $\mathrm{CIII}$, because $\mathrm{Cl}, \mathrm{Cll}$ and $\mathrm{Cll}$-like auxiliary enzymes can all donate electrons to $Q$ to form $\mathrm{QH}_{2}$, but $\mathrm{QH}_{2}$ can only deliver electrons to CIII. Thus, the allocation of $Q$ pool is of vital importance in regulating different metabolism pathway (Enriquez, 2016; Moreno-Loshuertos and Enriquez, 2016). One group estimated that $15 \%$ to $30 \%$ of the total $Q$ molecules were bound to proteins and the remaining fraction was likely to be free in membrane (Lass and Sohal, 1999; Lass et al., 1999; Lass et al., 1999). In 2008, Rossigol and his colleagues demonstrated that a portion of the total $Q$ pool was not able to be used for succinate oxidation (CII function). They estimated that the portion is $79 \%$ in muscle mitochondria and $21 \%$ in liver mitochondria (Benard et al., 2008). In 2013, Lapuente and his colleagues found that when the amount of $\mathrm{CIII}$ is less than the amount of $\mathrm{Cl}$, oxidation of succinate and glycerol-3-phosphate is blocked but oxidation of NADH remain unchanged (Lapuente-Brun et al., 2013). These results can be explained that most $\mathrm{CIII}$ is bound to $\mathrm{Cl}$ to form SC, and no free CIII is available to utilize the membrane $Q$ pool rather than the $Q$ pool segmented by SC. In 2014, Hirst's group reported that the activity of CIII can be maximized only when succinate and NADH are provided simultaneously, instead of either substrate alone (Blaza et al., 2014). In 2015, Anderson and his colleagues found that the impact of shortage of ubiquinone on different substrate respiration can vary. Oxidation of glycerol-3-phosphate is mostly affected, followed by succinate, and the oxidation of $\mathrm{NADH}$ is the least affected (Anderson et al., 2015) All these results suggest segmentation of $Q$ pools, but the structure basis in SC is not clear.

On the contrary, evidence for cytochrome c pool segmentation in SC between CIII and CIV are comparatively rare. The lacking of evidence for cytochrome $c$ pool segmentation in SC may be due to the fact that most CIV are in the free form (Genova, 2014). Even if there is actually a cyt.c pool segmentation in SC, the portion will be very small and not easy to detect. But according to our latest structure of respirasome, we provided a model showing that the binding site of Cyt.c in CIII and CIV are located near each other within $10 \mathrm{~nm}$ in the IMS plane formed by $\mathrm{TMH}$ ends of $\mathrm{Cl}$, CIII and CIV, and this arrangement can provide the possible structural basis for Cyt.c channeling (Gu et al., 2016).

\section{The organization of the respiratory chain}

Two models describing how the respiratory chain is organized are presented above, the fluid model (Hochli and Hackenbrock, 1976; Chazotte and Hackenbrock, 1991; Hackenbrock et al., 1984; Gupte et al., 1984; Schneider et al., 1980; Schneider et al., 1982; Schneider et al., 1982; Hackenbrock et al., 1980; Hochli and Hackenbrock 1979) by Hackenbrock and the solid model (Schagger and Pfeiffer, 2000; Schagger, 2001; Schagger and Pfeiffer, 2001; Wittig et al., 2006; Chance, 1950; Keilin and Hartree, 1947) by Schägger. Both of these models have their reasonable parts and some other parts that are not in coordination with existing experiment results. Integrating the advantages of the two models above, Antonio and his colleagues proposed the plastic model (Acin-Perez et al., 2008; Lapuente-Brun et al., 2013; Moreno-Loshuertos and Enriquez, 2016; Enriquez and Lenaz, 2014; Acin-Perez et al., 2008; Cogliati et al., 2013). In his model, the organization of OXPHOS complexes is very flexible. Both the assembled SCs and free 
individual complexes can perform their function, with SCs being more efficient in energy generation and less active in ROS production. The degree of free complexes integrating into SCs is very likely under elaborate regulation, to accommodate to different demands of the cell environment (Sun et al, 2016; Wang et al., 2016). Different forms of SCs may participate in different metabolic pathways. $S \mathrm{SI}_{1} \mathrm{II}_{2} I \mathrm{~V}_{1}$ can only oxidize $\mathrm{NADH}$, which is the main product of glucose metabolism, while $\mathrm{SCIII}_{2} \mathrm{IV}_{1}$ can receive $\mathrm{QH}_{2}$ from $\mathrm{Cll}$, which can oxidize succinate (Genova and Lenaz, 2014).

Recent studies indeed provided evidence that SC distribution is correlated with cell conditions (Vartak et al., 2013; Lapuente-Brun et al., 2013; Ikeda et al., 2013). SCAF1, previously called Cox7RP, is the first assembly factor functions only in SC assembly and not required in assembly of individual complexes. SCAF1 is required for superassembly of CIII and CIV, and in mice mutants lacking active SCAF1 no SCs requiring direct interaction between CIII and CIV exist, with almost all CIVs remain single complex form, but in these mutants no major biological problems were caused, which suggests the SCs and free complexes both function normally with the mutants probably lacking some fine regulations of energy supplication.

Moreover, experiment evidence has been given that the shape and curvature of inner mitochondria membrane, which are very dynamic, can have significant influence on SC distribution (Cogliati et al., 2013; Liesa and Shirihai, 2013). Besides, in different tissues, different species, different environmental conditions and different ages, the distribution of SC can vary significantly (Ramirez-Aguilar et al., 2011; Gomez et al., 2009; Lapuente-Brun et al., 2013; Hofmann et al., 2012; Frenzel et al., 2010; Feng et al., 2016). These heterogeneity of SC distribution is in line with the plastic model, although the correlation between specific SC distribution types and specific cell conditions is not defined yet (Enriquez, 2016).

\section{The structure of respirasome}

Structural study of respiratory supercomplexes could be traced back to the year of 2005, when Dudkina firstly determined the structure of $\mathrm{SCl}_{1} \mathrm{Il}_{2}$ purified from Arabidopsis thaliana at a resolution of $18 \AA$ through the single-particle cryo-EM technology, (Dudkina et al., 2005) which has been proved very useful since all the later structures of supercomplexes are determined this way. The $\mathrm{SCl}_{1} \mathrm{III}_{2}$ they purified proved to be very stable, and when compared with the map of individual complexes from mammals, CIII in the Arabidopsis thaliana $\mathrm{SCl}_{1} \mathrm{Il}_{2}$ is somehow smaller, while $\mathrm{Cl}$ has extra knob-like protein densities attached to either side of the hydrophobic arm, which is a unique feature not seen in $\mathrm{Cl}$ from any other species, and these extra proteins are required for the formation of supercomplex, indicating their interactions with CIII. But due to the limited resolution, Dudkina were not able to recognize the cavity responsible for ubiquinone-channeling.
Then in 2007, Jesco provided a structural model of $\mathrm{SCIII}_{2} \mathrm{IV}_{1-2}$ from yeast mitochondria at a resolution of $15 \AA$ (Heinemeyer et al., 2007). In yeast where $\mathrm{Cl}$ is lacking, CIII and CIV can form very stable supercomplexes. In their structures, CIII dimer either binds to one CIV or two CIV at different sides, the total structure showing a two-fold symmetry. The $\mathrm{III}_{2} \mathrm{IV}$ structure is very likely to be the brick of respiratory string. They also found cytochrome $c$ bound to their structure, and they estimated that the distance between Cyt.c binding sites in CIII and CIV was less than $40 \AA$, which was different from that in mammals.

Several years later, two groups independently reported the structure of $\mathrm{SCl}_{1} \mathrm{II}_{2} \mathrm{IV}$ from bovine heart at the resolution of $22 \AA$ (Dudkina et al., 2011) and $19 \AA$ (Althoff et al., 2011) respectively in 2011. Despite the $36 \AA$ structure of bovine $S \mathrm{I}_{1} \mathrm{II}_{2} \mathrm{IV}_{1}$ solved by Schäfer in 2007 , (Schafer et al., 2006) through which the location of $\mathrm{Cl}, \mathrm{CIII}$ and CIV could not be precisely determined because of the low resolution, these are the first structures of supercomplex from mammals, through which the arrangement of individual complexes could be easily affirmed. Both these groups used X-ray structures of individual complexes to build the model, and they all calculated the distance of $Q$ binding sites between $\mathrm{Cl}$ and $\mathrm{CIII}$ as $13 \mathrm{~nm}$, the distance of Cyt.c binding sites between CIII and CIV as $10 \mathrm{~nm}$. But due to the limited resolution, detailed information about interactions among $\mathrm{Cl}$, CIII and CIV could not be extracted (Fig. 1).

Recently, our lab solved the structure of $\mathrm{SCl}_{1} \mathrm{III}_{2} \mathrm{IV}_{1}$ (respirasome) from porcine heart at an overall resolution of 5.4 $\AA$, with the resolution of both individual $\mathrm{Cl}$ and $\mathrm{Clll}$ within the respirasome reaching $3.96 \AA$ using sub-region refinement (Gu et al., 2016). Aided by the $3.96 \AA$ density map of $\mathrm{Cl}$, we were able to accurately assign all the 14 core subunits together with 20 supernumerary subunits, and build 17 additional backbone models into the unoccupied density. Totally, $77 \mathrm{TMHs}$ from $\mathrm{Cl}$ were identified. Our model of $\mathrm{Cl}$ was proved to be correct by the latest work performed by Zhu and Fiedorczuk (Zhu et al., 2016; Fiedorczuk et al., 2016). In the $3.96 \AA$ density map of CIII, the distances between $[2 \mathrm{Fe}-2 \mathrm{~S}]$ and heme $c_{1},[2 \mathrm{Fe}-2 \mathrm{~S}]$ and heme $b_{L}$ are 30 $\AA$ and $27 \AA$ respectively, which suggests the CIII in our structure is in the 'int' state. Unfortunately, no density for Cyt. $c$ was found in our map (Fig. 2).

When compared with $\mathrm{Cl}$ in the free form, the matrix arm of $\mathrm{Cl}$ and the distal end of Cl's membrane arm bend a little towards the central part of Cl's membrane arm in the respirasome, to better interact with CIII and CIV. CIII form a dimer in the respirasome, located at concave side of Cl's membrane arm. The Clll dimer has a two-fold axis, which is perpendicular to the membrane plane, with the matrix domains of CIIl protruding around $75 \AA$ out of the membrane and facing the matrix arm of Cl. CIV sits at the distal end of Cl's membrane arm, and likely due to its flexibility, its density map is of relatively low resolution. Altogether, $77 \mathrm{TMHs}$ from $\mathrm{Cl}, 26$ TMHs from CIII and $28 \mathrm{TMHs}$ from CIV were identified, and the TMHs of the three complexes form an enormous 
A
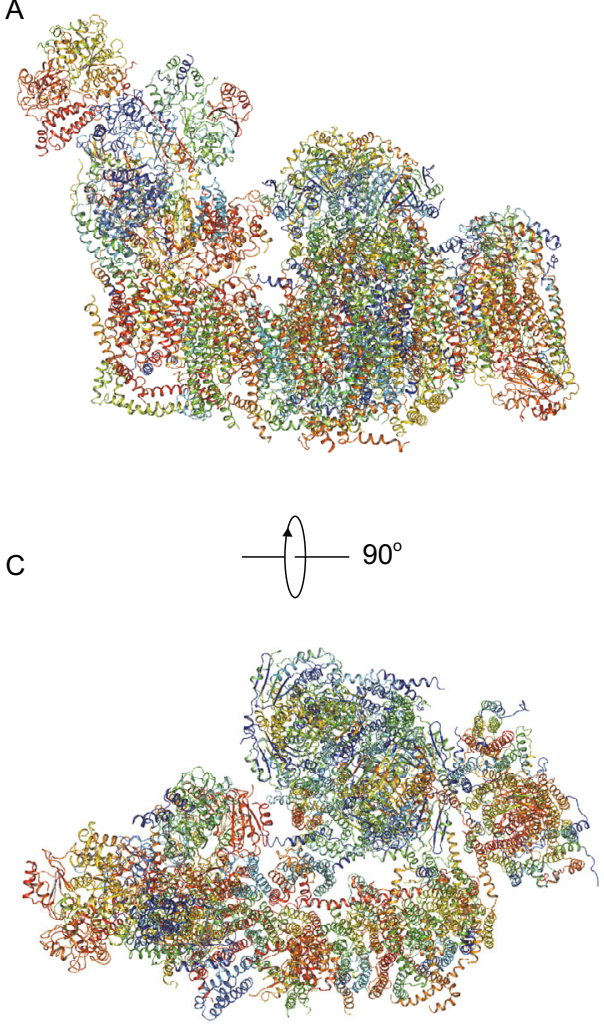

B

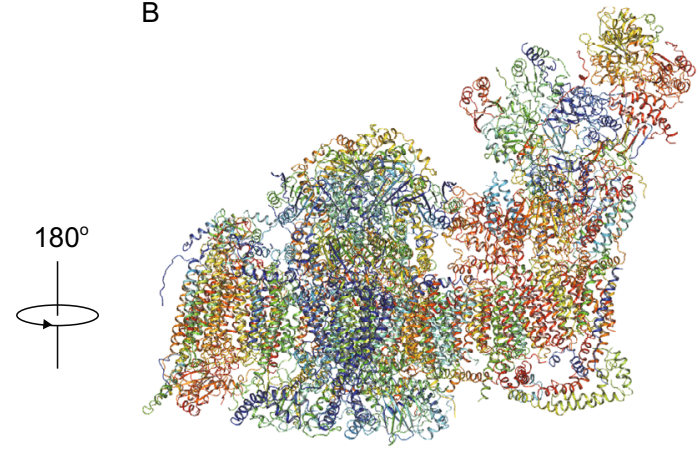

D $\quad \fallingdotseq-90^{\circ}$

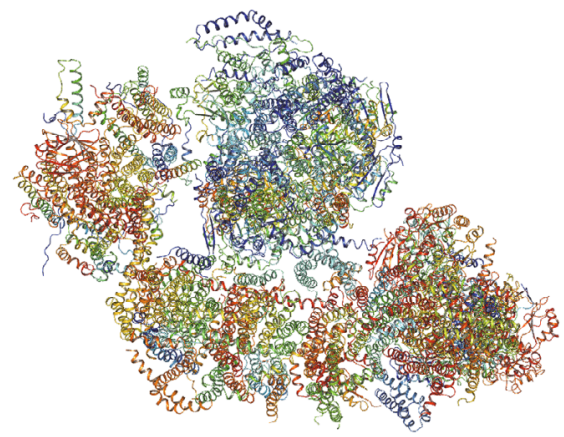

Figure 2. Overall structure of respirasome. The structure of respirasome from porcine heart at a resolution of $5.4 \AA$. (A) front view; (B) back view; (C) top view; (D) bottom view. The relations between the views are indicated by the angles.

transmembrane disk with their ends at the intermembrane side aligned to form a plane (Gu et al., 2016).

The interaction between $\mathrm{Cl}$ and $\mathrm{CIII}$ is rather stable. Within our structure, we can affirm that NDUFA11 of $\mathrm{Cl}$ directly interact with both $\mathrm{Cl}$ and CIII. The central part of NDUFA11 contains $4 \mathrm{TMHs}$, with an extra short helix at the $\mathrm{N}$-terminus and a loop region at the $\mathrm{C}$-terminus. The central TMHs of NDUFA11 bundle together and parallel the $\mathrm{TMH}$ domain of Cl's membrane arm. At one side, the $\mathrm{N}$-terminus of NDUFA11 interacts with ND5, the C-terminal loop contacts $\mathrm{ND} 2$, and $\mathrm{TMH} 4$ interacts with ND4. At the other side, NDUFA11 is close to UQCRB and UQCRQ of one CIII in the dimer. These features agree with previous reports that a mutation disrupting TMH1 of NDUFA11 destabilized the entire complex, and that blockof NDUFA11 expression disrupted the assembly of $\mathrm{Cl}$, resultingin the accumulation of $550 \mathrm{kDa}$ and $815 \mathrm{kDa} \mathrm{Cl}$ sub-complexes. At another site, the $\mathrm{N}$-lobe of NDUFB9 glues $\mathrm{Cl}$ and $\mathrm{CIII}$ together. A short loop of UQCRC1 (subunit of CIII) inserts into the groove circled by the N-lobe of NDUFB9 and an unassigned subunit of $\mathrm{Cl}$, while the LYR-motif in the N-lobe of NDUFB9 is important for NDUFAB1 (subunit of $\mathrm{Cl}$ ) binding. These features are in accordance with previous data that mutations in NDUFB9 can lead to $\mathrm{Cl}$ deficiency, $\mathrm{NAD}^{+} / \mathrm{NADH}$ imbalance and tumor metastasis. Gene blast shows the binding motifs in NDUFA11, NDUFB9 and UQCRC1 are highly conserved across species, suggesting their important roles in supercomplex formation (Gu et al., 2016).

CIV binds relatively loosely to $\mathrm{Cl}$ and $\mathrm{CIII}$. The TMH of COX7C (subunit of CIV) is packed against the last $\mathrm{TMH}$ of ND5 at the distal end of Cl's membrane arm. Additionally, at the matrix side, the flexible region of COX7A (subunit of CIV) contact with both UQCRC1 and UQCR11 (subunits of CIII). Perhaps due to the lack of supernumerary subunits from $\mathrm{Cl}$ functioning as bridge, these interactions are slightly weaker compared to $\mathrm{Cl}-\mathrm{CIII}$ interaction. Furthermore, there are clear gaps between these three complexes. According to multiple previous reports, a large amount of different lipid molecules, including cardiolipin, phosphotidylcholine, and phosphotidylethanolamine, should be present in the isolated samples, we propose these lipid molecules may occupy these gaps to further stabilize the respirasome (Gu et al., 2016).

Our structure provides evidence for substrate channeling. Ubiquinone is supposed to be trapped in the gap between $\mathrm{Cl}$ and CIII, which is filled with lipid molecules, and this compartmental arrangement can greatly increase the efficiency 
of ubiquinone transport. At the IMS side, the binding site of Cyt.c in CIII and CIV are located near each other within 10 $\mathrm{nm}$ in the plane formed by $\mathrm{TMH}$ ends of $\mathrm{CI}, \mathrm{CIII}$ and $\mathrm{CIV}$. This feature vastly facilitates Cyt.c communicating between CIII and CIV (Gu et al., 2016) (Fig. 2).

\section{Evidence for respiratory strings or megacomplexes}

Cryo-electron tomography and freeze-fracture EM studies in earlier years indicate the respiratory complexes could form higher order structures which might even influence the shape of mitochondria cristae (Allen et al., 1989) Richard in 1989 reported that in his fracture photos, ATPase could be seen clearly form dimers, with some other large particles in accordance with complex I (now considered the supercomplexes) regularly arrayed in rows, and many other authors showed similar pictures (Heinemeyer et al., 2007; Nicastro et al., 2000; Strauss et al., 2008; Sousa et al., 2013). The ATPase dimers in these electron microscopy photographs were somehow far more obvious than the respiratory rows, and that's the reason why investigations into megacomplexes proved to be difficult. Years later, Schägger measured the size of individual $\mathrm{Cl}, \mathrm{CIII}$, $\mathrm{CIV}$, and the distance between particles in the presumed respiratory row, titrated the ratio of $\mathrm{Cl}$ :CIII:CIV in supercomplexes, and then proposed the concept of respiratory string, where supercomplex $I_{1} \|_{2} I V_{4}$ is the brick, forming the dimer first and then a string linked by CIV (Wittig et al., 2006). After that, we and many others identified higher molecular weight bands than the bands of $\mathrm{SCl}_{1} \mathrm{II}_{2} \mathrm{IV}_{1}$ in BNPG, and classified particles from Cryo-EM images similar to the presumed respiratory dimer, or megacomplex (Gu et al., 2016; Heinemeyer et al., 2007; Sousa et al., 2013; Bultema et al., 2009; Davies et al., 2011). These results were obtained from different organisms, including porcine, potato, yeast and bacteria, suggesting the existence of a higher order arrangement of respiratory chain elements across species. Due to its large scale and highly compacted organization, respiratory string could be responsible for stabilizing the cristae structure and function very efficiently in urgent conditions (Riva et al., 2003, 2006; Perkins et al., 1997; Muhleip et al., 2016; Stroud and Ryan, 2013; Barcena et al., 2010; Chen et al., 2015).

\section{CONCLUSIONS AND PERSPECTIVES}

Researches focusing on the energy metabolism have always been of great importance and interest, and after a century's effort, scientists have got exciting achievements in understanding the respiratory chain. We know that the respiratory chain elements are arranged in a highly ordered manner, and have reached some reasonable speculations about how these gigantic protein machines work properly at an atomic level, but some critical information is still lacking. For example, the organization of respiratory chain can vary to adapt to different cell conditions, but the accurate regulatory mechanism is far from clear; in addition, accumulating evidence is indicating the existence of megacomplexes, which we know very little about besides assuming it could influence the shape of mitochondria and might function in urgent situations; moreover, in order to provide valuable information for clinical application, the structure of respirasome from human, rather than bovine or porcine is required. Massive efforts are still needed in understanding the details of cellular respiration and developing remedy plans for diseases relating to mitochondria abnormality. We believe a more delicate picture depicting the detailed information of respirasome is on its way.

\section{ACKNOWLEDGEMENTS}

This work was supported by funds from the National Basic Research Program (973 Program) (Nos. 2016YFA0501101 and 2012CB911101), and the National Science Fund for Distinguished Young Scholars (No. 3163000168) and the National Natural Science Foundation of China (Grant Nos. 31030020 and 31170679).

\section{COMPLIANCE WITH ETHICS GUIDELINES}

RunyuGuo, JinkeGu, Meng Wu, andMaojun Yang declare that they have no conflict of interest.

This article does not contain any studies with human or animal subjects performed by any of the authors.

\section{OPEN ACCESS}

This article is distributed under the terms of the Creative Commons Attribution 4.0 International License (http://creativecommons.org/ licenses/by/4.0/), which permits unrestricted use, distribution, and reproduction in any medium, provided you give appropriate credit to the original author(s) and the source, provide a link to the Creative Commons license, and indicate if changes were made.

\section{REFERENCES}

Acin-Perez R, Enriquez JA (2014) The function of the respiratory supercomplexes: the plasticity model. Biochim Biophys Acta 1837:444-450. doi:10.1016/j.bbabio.2013.12.009

Acin-Perez R et al (2004) Respiratory complex III is required to maintain complex I in mammalian mitochondria. Mol Cell 13:805815

Acin-Perez R, Fernandez-Silva P, Peleato ML, Perez-Martos A, Enriquez JA (2008) Respiratory active mitochondrial supercomplexes. Mol Cell 32:529-539. doi:10.1016/j.molcel.2008.10.021

Allen RD, Schroeder CC, Fok AK (1989) An investigation of mitochondrial inner membranes by rapid-freeze deep-etch techniques. J Cell Biol 108:2233-2240

Althoff T, Mills DJ, Popot JL, Kuhlbrandt W (2011) Arrangement of electron transport chain components in bovine mitochondrial supercomplex I1IIIIV1. EMBO J30:4652-4664. doi:10.1038/ emboj.2011.324

Anderson CM et al (2015) Dependence of brown adipose tissue function on CD36-mediated coenzyme Q uptake. Cell Rep 10:505-515. doi:10.1016/j.celrep.2014.12.048 
Baradaran R, Berrisford JM, Minhas GS, Sazanov LA (2013) Crystal structure of the entire respiratory complex I. Nature 494:443-448. doi:10.1038/nature11871

Baranova EA, Holt PJ, Sazanov LA (2007) Projection structure of the membrane domain of Escherichia coli respiratory complex I at $8 \mathrm{~A}$ resolution. J Mol Biol 366:140-154. doi:10.1016/j.jmb.2006.11.026

Barcena C, Martinez MA, Ortega MP, Munoz HG, Sarraga GU (2010) Mitochondria with tubulovesicular cristae in renal oncocytomas. Ultrastruct Pathol 34:315-320. doi:10.3109/01913123. 2010.506021

Benard G et al (2008) Functional dynamic compartmentalization of respiratory chain intermediate substrates: implications for the control of energy production and mitochondrial diseases. Int $\mathrm{J}$ Biochem Cell Biol 40:1543-1554. doi:10.1016/j.biocel.2007.11. 023

Berrisford JM, Sazanov LA (2009) Structural basis for the mechanism of respiratory complex I. J Biol Chem 284:29773-29783. doi:10.1074/jbc.M109.032144

Blaza JN, Serreli R, Jones AJ, Mohammed K, Hirst J (2014) Kinetic evidence against partitioning of the ubiquinone pool and the catalytic relevance of respiratory-chain supercomplexes. Proc Natl Acad Sci U S A 111:15735-15740. doi:10.1073/pnas. 1413855111

Bultema JB, Braun HP, Boekema EJ, Kouril R (2009) Megacomplex organization of the oxidative phosphorylation system by structural analysis of respiratory supercomplexes from potato. Biochim Biophys Acta 1787:60-67. doi:10.1016/j.bbabio.2008.10.010

Chance B (1950) Oxidase activity-light absorption relationships in cytochrome system of heart muscle preparations. Biol Bull 99:318

Chance B, Estabrook RW, Lee CP (1963) Electron Transport in the Oxysome. Science 140:379-380. doi:10.1126/science.140.3565. 379-c

Chazotte B, Hackenbrock CR (1991) Lateral diffusion of redox components in the mitochondrial inner membrane is unaffected by inner membrane folding and matrix density. J Biol Chem 266:5973-5979

Chen C, Chen Y, Guan MX (2015) A peep into mitochondrial disorder: multifaceted from mitochondrial DNA mutations to nuclear gene modulation. Protein Cell 6:862-870. doi:10.1007/ s13238-015-0175-z

Cogliati S et al (2013) Mitochondrial cristae shape determines respiratory chain supercomplexes assembly and respiratory efficiency. Cell 155:160-171. doi:10.1016/j.cell.2013.08.032

Cogliati S, Enriquez JA, Scorrano L (2016) Mitochondrial Cristae: Where Beauty Meets Functionality. Trends Biochem Sci 41:261273. doi:10.1016/j.tibs.2016.01.001

Daoud R, Forget L, Lang BF (2012) Yeast mitochondrial RNase P, RNase $Z$ and the RNA degradosome are part of a stable supercomplex. Nucleic Acids Res 40:1728-1736. doi:10.1093/nar/ gkr941

Davies KM et al (2011) Macromolecular organization of ATP synthase and complex I in whole mitochondria. Proc Natl Acad Sci USA 108:14121-14126. doi:10.1073/pnas.1103621108

Dekker JP, Boekema EJ (2005) Supramolecular organization of thylakoid membrane proteins in green plants. Biochim Biophys Acta 1706:12-39. doi:10.1016/j.bbabio.2004.09.009
Diaz F, Fukui H, Garcia S, Moraes CT (2006) Cytochrome c oxidase is required for the assembly/stability of respiratory complex $\mathrm{I}$ in mouse fibroblasts. Mol Cell Biol 26:4872-4881. doi:10.1128/ MCB.01767-05

Dudkina NV, Eubel H, Keegstra W, Boekema EJ, Braun HP (2005) Structure of a mitochondrial supercomplex formed by respiratorychain complexes I and III. Proc Natl Acad Sci USA 102:32253229. doi:10.1073/pnas.0408870102

Dudkina NV, Heinemeyer J, Sunderhaus S, Boekema EJ, Braun HP (2006) Respiratory chain supercomplexes in the plant mitochondrial membrane. Trends Plant Sci 11:232-240. doi:10.1016/j. tplants.2006.03.007

Dudkina NV, Kudryashev M, Stahlberg H, Boekema EJ (2011) Interaction of complexes I, III, and IV within the bovine respirasome by single particle cryoelectron tomography. Proc Natl Acad Sci USA 108:15196-15200. doi:10.1073/pnas.1107819108

Dudkina NV, Folea IM, Boekema EJ (2015) Towards structural and functional characterization of photosynthetic and mitochondrial supercomplexes. Micron 72:39-51. doi:10.1016/j.micron.2015. 03.002

Efremov RG, Sazanov LA (2011) Structure of the membrane domain of respiratory complex I. Nature 476:414-420. doi:10.1038/ nature 10330

Efremov RG, Baradaran R, Sazanov LA (2010) The architecture of respiratory complex I. Nature 465:441-445. doi:10.1038/ nature09066

Enriquez JA (2016) Supramolecular organization of respiratory complexes. Annu Rev Physiol 78:533-561. doi:10.1146/ annurev-physiol-021115-105031

Enriquez JA, Lenaz G (2014) Coenzyme q and the respiratory chain: coenzyme q pool and mitochondrial supercomplexes. Mol Syndromol 5:119-140. doi:10.1159/000363364

Ernster L, Schatz G (1981) Mitochondria: a historical review. J Cell Biol 91:227s-255s

Eubel H, Jansch L, Braun HP (2003) New insights into the respiratory chain of plant mitochondria. Supercomplexes and a unique composition of complex II. Plant Physiol 133:274-286

Eubel H, Heinemeyer J, Sunderhaus S, Braun HP (2004) Respiratory chain supercomplexes in plant mitochondria. Plant Physiol Biochem 42:937-942. doi:10.1016/j.plaphy.2004.09.010

Feng J, Lu S, Ding Y, Zheng M, Wang X (2016) Homocysteine activates $T$ cells by enhancing endoplasmic reticulum-mitochondria coupling and increasing mitochondrial respiration. Protein Cell 7:391-402. doi:10.1007/s13238-016-0245-x

Fiedorczuk K et al (2016) Atomic structure of the entire mammalian mitochondrial complex I. Nature. doi:10.1038/nature19794

Frenzel M, Rommelspacher H, Sugawa MD, Dencher NA (2010) Ageing alters the supramolecular architecture of OxPhos complexes in rat brain cortex. Exp Gerontol 45:563-572. doi:10.1016/ j.exger.2010.02.003

Gao X et al (2002) The crystal structure of mitochondrial cytochrome bc1 in complex with famoxadone: the role of aromatic-aromatic interaction in inhibition. Biochemistry 41:11692-11702

Gao X et al (2003) Structural basis for the quinone reduction in the bc1 complex: a comparative analysis of crystal structures of mitochondrial cytochrome bc1 with bound substrate and inhibitors at the Qi site. Biochemistry 42:9067-9080. doi:10.1021/bi0341814 
Genova ML (2014) Electron Transport in the Mitochondrial Respiratory Chain. Struct Basis Biol Energy Gener 39:401-417. doi:10. 1007/978-94-017-8742-0_21

Genova ML, Lenaz G (2014) Functional role of mitochondrial respiratory supercomplexes. Biochim Biophys Acta 1837:427443. doi:10.1016/j.bbabio.2013.11.002

Gomez LA, Monette JS, Chavez JD, Maier CS, Hagen TM (2009) Supercomplexes of the mitochondrial electron transport chain decline in the aging rat heart. Arch Biochem Biophys 490:30-35. doi:10.1016/j.abb.2009.08.002

Green DE, Tzagoloff A (1966) The mitochondrial electron transfer chain. Arch Biochem Biophys 116:293-304

$\mathrm{Gu} \mathrm{J}$ et al (2016) The architecture of the mammalian respirasome. Nature 537(7622):639-643. doi:10.1038/nature19359

Gupte $S$ et al (1984) Relationship between lateral diffusion, collision frequency, and electron transfer of mitochondrial inner membrane oxidation-reduction components. Proc Natl Acad Sci USA 81:2606-2610

Hackenbrock CR, Schneider H, Lemasters JJ, Hochli M (1980) Relationships between bilayer lipid, motional freedom of oxidoreductase components, and electron transfer in the mitochondrial inner membrane. Adv Exp Med Biol 132:245-263

Hackenbrock CR, Gupte S, Wu ES, Jacobson K (1984) Lateral diffusion, collision and efficiency of oxidation-reduction components in mitochondrial electron transport. Biochem Soc Trans 12:402-403

Hackenbrock CR, Chazotte B, Gupte SS (1986) The random collision model and a critical assessment of diffusion and collision in mitochondrial electron transport. $\mathrm{J}$ Bioenerg Biomembr 18:331-368

Hatefi Y, Haavik AG, Fowler LR, Griffiths DE (1962) Studies on the electron transfer system. XLII. Reconstitution of the electron transfer system. J Biol Chem 237:2661-2669

Heinemeyer J, Braun HP, Boekema EJ, Kouril R (2007) A structural model of the cytochrome $C$ reductase/oxidase supercomplex from yeast mitochondria. J Biol Chem 282:12240-12248. doi:10. 1074/jbc.M610545200

Hochli M, Hackenbrock CR (1976) Fluidity in mitochondrial membranes: thermotropic lateral translational motion of intramembrane particles. Proc Natl Acad Sci USA 73:1636-1640

Hochli M, Hackenbrock CR (1979) Lateral translational diffusion of cytochrome $c$ oxidase in the mitochondrial energy-transducing membrane. Proc Natl Acad Sci USA 76:1236-1240

Hofmann AD et al (2012) OXPHOS supercomplexes as a hallmark of the mitochondrial phenotype of adipogenic differentiated human MSCs. PLoS One 7:e35160. doi:10.1371/journal.pone. 0035160

Huang LS et al (2006) 3-nitropropionic acid is a suicide inhibitor of mitochondrial respiration that, upon oxidation by complex II, forms a covalent adduct with a catalytic base arginine in the active site of the enzyme. J Biol Chem 281:5965-5972. doi:10. 1074/jbc.M511270200

Ikeda K, Shiba S, Horie-Inoue K, Shimokata K, Inoue S (2013) A stabilizing factor for mitochondrial respiratory supercomplex assembly regulates energy metabolism in muscle. Nat Commun 4:2147. doi:10.1038/ncomms3147
Iwata S, Ostermeier C, Ludwig B, Michel H (1995) Structure at 2.8 A resolution of cytochrome $\mathrm{C}$ oxidase from Paracoccus denitrificans. Nature 376:660-669. doi:10.1038/376660a0

Iwata $S$ et al (1998) Complete structure of the 11-subunit bovine mitochondrial cytochrome bc1 complex. Science 281:64-71

Johnson GN (2011) Physiology of PSI cyclic electron transport in higher plants. Biochim Biophys Acta 1807:384-389. doi:10.1016/ j.bbabio.2010.11.009

Kaila VR, Sharma V, Wikstrom M (2011) The identity of the transient proton loading site of the proton-pumping mechanism of cytochrome c oxidase. Biochim Biophys Acta 1807:80-84. doi:10.1016/j.bbabio.2010.08.014

Kannt A, Lancaster CR, Michel H (1998) The coupling of electron transfer and proton translocation: electrostatic calculations on Paracoccus denitrificans cytochrome c oxidase. Biophys J74:708-721. doi:10.1016/S0006-3495(98)73996-7

Keilin D, Hartree EF (1947) Activity of the cytochrome system in heart muscle preparations. Biochem J41:500-502

Kouril R, Dekker JP, Boekema EJ (2012) Supramolecular organization of photosystem II in green plants. Biochim Biophys Acta 1817:2-12. doi:10.1016/j.bbabio.2011.05.024

Lamantea $E$ et al (2002) A novel nonsense mutation (Q352X) in the mitochondrial cytochrome $b$ gene associated with a combined deficiency of complexes I and III. Neuromuscul Disord 12:49-52

Lange C, Hunte C (2002) Crystal structure of the yeast cytochrome bc1 complex with its bound substrate cytochrome c. Proc Natl Acad Sci USA 99:2800-2805. doi:10.1073/pnas.052704699

Lapuente-Brun E et al (2013) Supercomplex assembly determines electron flux in the mitochondrial electron transport chain. Science 340:1567-1570. doi:10.1126/science.1230381

Lass A, Sohal RS (1999) Comparisons of coenzyme Q bound to mitochondrial membrane proteins among different mammalian species. Free Radic Biol Med 27:220-226

Lass A, Kwong L, Sohal RS (1999a) Mitochondrial coenzyme Q content and aging. Biofactors 9:199-205

Lass A, Forster MJ, Sohal RS (1999b) Effects of coenzyme Q10 and alpha-tocopherol administration on their tissue levels in the mouse: elevation of mitochondrial alpha-tocopherol by coenzyme Q10. Free Radic Biol Med 26:1375-1382

Lenaz G, Genova ML (2010) Structure and organization of mitochondrial respiratory complexes: a new understanding of an old subject. Antioxid Redox Signal 12:961-1008. doi:10.1089/ ars.2009.2704

Liao $Y$ et al (2015) Mitochondrial calcium uniporter protein MCU is involved in oxidative stress-induced cell death. Protein Cell 6:434-442. doi:10.1007/s13238-015-0144-6

Liesa M, Shirihai OS (2013) Mitochondrial dynamics in the regulation of nutrient utilization and energy expenditure. Cell Metab 17:491506. doi:10.1016/j.cmet.2013.03.002

Livingston AK, Cruz JA, Kohzuma K, Dhingra A, Kramer DM (2010) An Arabidopsis mutant with high cyclic electron flow around photosystem I (hcef) involving the NADPH dehydrogenase complex. Plant Cell 22:221-233. doi:10.1105/tpc.109. 071084

Maranzana E, Barbero G, Falasca Al, Lenaz G, Genova ML (2013) Mitochondrial respiratory supercomplex association limits 
production of reactive oxygen species from complex I. Antioxid Redox Signal 19:1469-1480. doi:10.1089/ars.2012.4845

Mitchell P (1961) Coupling of phosphorylation to electron and hydrogen transfer by a chemi-osmotic type of mechanism. Nature 191:144-148

Moreno-Lastres D et al (2012) Mitochondrial complex I plays an essential role in human respirasome assembly. Cell Metab 15:324-335. doi:10.1016/j.cmet.2012.01.015

Moreno-Loshuertos R, Enriquez JA (2016) Respiratory supercomplexes and the functional segmentation of the CoQ pool. Free Radic Biol Med. doi:10.1016/j.freeradbiomed.2016.04.018

Morgan DJ, Sazanov LA (2008) Three-dimensional structure of respiratory complex I from Escherichia coli in ice in the presence of nucleotides. Biochim Biophys Acta 1777:711-718. doi:10. 1016/j.bbabio.2008.03.023

Muhleip AW et al (2016) Helical arrays of U-shaped ATP synthase dimers form tubular cristae in ciliate mitochondria. Proc Natl Acad Sci U S A 113:8442-8447. doi:10.1073/pnas.1525430113

Nicastro D, Frangakis AS, Typke D, Baumeister W (2000) Cryoelectron tomography of neurospora mitochondria. J Struct Biol 129:48-56. doi:10.1006/jsbi.1999.4204

Nubel E, Wittig I, Kerscher S, Brandt U, Schagger H (2009) Twodimensional native electrophoretic analysis of respiratory supercomplexes from Yarrowia lipolytica. Proteomics 9:2408-2418. doi:10.1002/pmic.200800632

Ostermeier C, Harrenga A, Ermler U, Michel H (1997) Structure at 2.7 A resolution of the Paracoccus denitrificans two-subunit cytochrome c oxidase complexed with an antibody FV fragment. Proc Natl Acad Sci U S A 94:10547-10553

Palsdottir H, Lojero CG, Trumpower BL, Hunte C (2003) Structure of the yeast cytochrome bc1 complex with a hydroxyquinone anion Qo site inhibitor bound. J Biol Chem 278:31303-31311. doi:10. 1074/jbc.M302195200

Perkins $\mathrm{G}$ et al (1997) Electron tomography of neuronal mitochondria: three-dimensional structure and organization of cristae and membrane contacts. J Struct Biol 119:260-272. doi:10.1006/jsbi. 1997.3885

Pfeiffer K et al (2003) Cardiolipin stabilizes respiratory chain supercomplexes. J Biol Chem 278:52873-52880. doi:10.1074/ jbc.M308366200

Ramirez-Aguilar SJ et al (2011) The composition of plant mitochondrial supercomplexes changes with oxygen availability. J Biol Chem 286:43045-43053. doi:10.1074/jbc.M111.252544

Riva A, Loffredo F, Uccheddu A, Riva FT, Tandler B (2003) Mitochondria of human adrenal cortex have tubular cristae with bulbous tips. J Clin Endocrinol Metab 88:1903-1906. doi:10. 1210/jc.2002-030013

Riva A et al (2006) Structure of cristae in cardiac mitochondria of aged rat. Mech Ageing Dev 127:917-921. doi:10.1016/j.mad. 2006.09.002

Rochaix JD (2014) Regulation and dynamics of the light-harvesting system. Annu Rev Plant Biol 65:287-309. doi:10.1146/annurevarplant-050213-040226

Sazanov LA (2007) Respiratory complex I: mechanistic and structural insights provided by the crystal structure of the hydrophilic domain. Biochemistry 46:2275-2288. doi:10.1021/bi602508x
Sazanov LA (2015) A giant molecular proton pump: structure and mechanism of respiratory complex I. Nat Rev Mol Cell Biol 16:375-388. doi:10.1038/nrm3997

Sazanov LA, Hinchliffe P (2006) Structure of the hydrophilic domain of respiratory complex I from Thermus thermophilus. Science 311:1430-1436. doi:10.1126/science.1123809

Schafer $E$ et al (2006) Architecture of active mammalian respiratory chain supercomplexes. J Biol Chem 281:15370-15375. doi:10. 1074/jbc.M513525200

Schagger H (2001) Respiratory chain supercomplexes. IUBMB Life 52:119-128. doi:10.1080/15216540152845911

Schagger H (2002) Respiratory chain supercomplexes of mitochondria and bacteria. Biochim Biophys Acta 1555:154-159

Schagger H, Pfeiffer K (2000) Supercomplexes in the respiratory chains of yeast and mammalian mitochondria. EMBO J19:17771783. doi:10.1093/emboj/19.8.1777

Schagger H, Pfeiffer K (2001) The ratio of oxidative phosphorylation complexes I-V in bovine heart mitochondria and the composition of respiratory chain supercomplexes. J Biol Chem 276:3786137867. doi:10.1074/jbc.M106474200

Schneider H, Lemasters JJ, Hochli M, Hackenbrock CR (1980) Liposome-mitochondrial inner membrane fusion. Lateral diffusion of integral electron transfer components. J Biol Chem 255:3748-3756

Schneider H, Lemasters JJ, Hackenbrock CR (1982a) Lateral diffusion of ubiquinone during electron transfer in phospholipidand ubiquinone-enriched mitochondrial membranes. J Biol Chem 257:10789-10793

Schneider H, Hochli M, Hackenbrock CR (1982b) Relationship between the density distribution of intramembrane particles and electron transfer in the mitochondrial inner membrane as revealed by cholesterol incorporation. J Cell Biol 94:387-393

Sousa PM et al (2013) The bc:caa3 supercomplexes from the Gram positive bacterium Bacillus subtilis respiratory chain: a megacomplex organization? Arch Biochem Biophys 537:153-160. doi:10.1016/j.abb.2013.07.012

Strauss M, Hofhaus G, Schroder RR, Kuhlbrandt W (2008) Dimer ribbons of ATP synthase shape the inner mitochondrial membrane. EMBO J27:1154-1160. doi:10.1038/emboj.2008.35

Stroh A et al (2004) Assembly of respiratory complexes I, III, and IV into NADH oxidase supercomplex stabilizes complex I in Paracoccus denitrificans. J Biol Chem 279:5000-5007. doi:10.1074/ jbc.M309505200

Stroud DA, Ryan MT (2013) Mitochondria: organization of respiratory chain complexes becomes cristae-lized. Curr Biol 23:R969R971. doi:10.1016/j.cub.2013.09.035

Stuart RA (2008) Supercomplex organization of the oxidative phosphorylation enzymes in yeast mitochondria. J Bioenerg Biomembr 40:411-417. doi:10.1007/s10863-008-9168-4

Sun F et al (2005) Crystal structure of mitochondrial respiratory membrane protein complex II. Cell 121:1043-1057. doi:10.1016/ j.cell.2005.05.025

Sun D, Li B, Quu R, Fang H, Lyu J (2016) Cell Type-Specific Modulation of Respiratory Chain Supercomplex Organization. Int J Mol Sci. doi:10.3390/ijms17060926

Tsukihara T et al (1995) Structures of metal sites of oxidized bovine heart cytochrome c oxidase at 2.8 A. Science 269:1069-1074 
Tsukihara $T$ et al (1996) The whole structure of the 13-subunit oxidized cytochrome $\mathrm{c}$ oxidase at $2.8 \mathrm{~A}$. Science $272: 1136-1144$

Tsukihara Tet al (2003) The low-spin heme of cytochrome c oxidase as the driving element of the proton-pumping process. Proc Natl Acad Sci U S A 100:15304-15309. doi:10.1073/pnas.263 5097100

Vartak R, Porras CA, Bai Y (2013) Respiratory supercomplexes: structure, function and assembly. Protein Cell 4:582-590. doi:10. 1007/s13238-013-3032-y

Vempati UD, Han X, Moraes CT (2009) Lack of cytochrome c in mouse fibroblasts disrupts assembly/stability of respiratory complexes I and IV. J Biol Chem 284:4383-4391. doi:10.1074/jbc. M805972200

Vinothkumar KR, Zhu J, Hirst J (2014) Architecture of mammalian respiratory complex I. Nature 515:80-84. doi:10.1038/nature 13686

Wang X et al (2016) Overexpression of SIRT3 disrupts mitochondrial proteostasis and cell cycle progression. Protein Cell 7:295-299. doi:10.1007/s13238-016-0251-z

Wenz T et al (2009) Role of phospholipids in respiratory cytochrome bc(1) complex catalysis and supercomplex formation. Biochim Biophys Acta 1787:609-616. doi:10.1016/j.bbabio.2009.02.012
Wittig I, Braun HP, Schagger H (2006a) Blue native PAGE. Nat Protoc 1:418-428. doi:10.1038/nprot.2006.62

Wittig I, Carrozzo R, Santorelli FM, Schagger H (2006b) Supercomplexes and subcomplexes of mitochondrial oxidative phosphorylation. Biochim Biophys Acta 1757:1066-1072. doi:10.1016/j. bbabio.2006.05.006

Xia D et al (1997) Crystal structure of the cytochrome bc1 complex from bovine heart mitochondria. Science 277:60-66

Yankovskaya V et al (2003) Architecture of succinate dehydrogenase and reactive oxygen species generation. Science 299:700704. doi:10.1126/science.1079605

Yoshikawa S et al (1998) Redox-coupled crystal structural changes in bovine heart cytochrome c oxidase. Science 280:1723-1729

Zhang $Z$ et al (1998) Electron transfer by domain movement in cytochrome bc1. Nature 392:677-684. doi:10.1038/33612

Zhang M, Mileykovskaya E, Dowhan W (2005) Cardiolipin is essential for organization of complexes III and IV into a supercomplex in intact yeast mitochondria. J Biol Chem 280:29403-29408. doi:10.1074/jbc.M504955200

Zhu J, Vinothkumar KR, Hirst J (2016) Structure of mammalian respiratory complex I. Nature 536:354-358. doi:10.1038/nature19095 